\title{
Do parents of children with attention-deficit/ hyperactivity disorder (ADHD) receive adequate information about the disorder and its treatments? A qualitative investigation
}

\author{
This article was published in the following Dove Press journal: \\ Patient Preference and Adherence \\ 8 May 2014 \\ Number of times this article has been viewed
}

\author{
Rana Ahmed' \\ Jacqueline M Borst ${ }^{2}$ \\ Cheng Wei Yong ${ }^{3}$ \\ Parisa Aslani' \\ 'Faculty of Pharmacy, The University \\ of Sydney, Sydney, NSW, Australia; \\ ${ }^{2}$ Department of Pharmaceutical \\ Sciences, Utrecht University, Utrecht, \\ the Netherlands; ${ }^{3}$ School of Pharmacy, \\ The University of Nottingham, \\ Nottingham, UK
}

\begin{abstract}
Background: Attention-deficit/hyperactivity disorder (ADHD) is the most prevalent pediatric neurodevelopmental condition, commonly treated using pharmacological agents such as stimulant medicines. The use of these agents remains contentious, placing parents in a difficult position when deciding to initiate and/or continue their child's treatment. Parents refer to a range of information sources to assist with their treatment decision-making. This qualitative study aimed to investigate 1) parents' ADHD-related knowledge pre- and post-diagnosis, 2) the information sources accessed by parents, 3 ) whether parents' information needs were met post-diagnosis, and 4) parents' views about strategies to meet their information needs.
\end{abstract}

Methods: Three focus groups ( $\mathrm{n}=16$ parents), each lasting $1.0-1.5$ hours were conducted. Focus groups were audio-recorded and transcribed verbatim. Transcripts were analyzed using the framework method, coded, and categorized into themes.

Results: Generally, parents had limited ADHD-related knowledge prior to their child's diagnosis and perceived prescription medicines indicated for ADHD in a negative context. Parents reported improved knowledge after their child's diagnosis; however, they expressed dissatisfaction with information that they accessed, which was often technical and not tailored to their child's needs. Verbal information sought from health care professionals was viewed to be reliable but generally medicine-focused and not necessarily comprehensive. Parents identified a need for concise, tailored information about ADHD, the medicines used for its treatment, and changes to their child's medication needs with age. They also expressed a desire for increased availability of support groups and tools to assist them in sourcing information from health care professionals during consultations, such as question prompt lists.

Conclusion: There are gaps in parents' knowledge about ADHD and its treatment, and an expressed need for tailored and reliable information. Future research needs to focus on providing parents with avenues to access concise, reliable, and relevant information and support in order to empower them to make the best treatment decision for their child.

Keywords: information needs, question prompt list

\section{Introduction}

Attention-deficit hyperactivity disorder (ADHD) was first identified over 100 years ago and was initially described as "hyperactivity" or the "hyperkinetic disorder of childhood". ${ }^{1}$ Today, this pediatric disorder is considered to be the most prevalent neurodevelopmental condition ${ }^{2,3}$ affecting between $3 \%$ and $7 \%$ of school-aged children. ${ }^{4}$ Affected children commonly display symptoms of inattention, hyperactivity, or impulsivity, ${ }^{5,6}$ which can impede their academic ${ }^{3,7}$ and social progress ${ }^{8,9}$ as well as
Correspondence: Rana Ahmed Room N502, Pharmacy Building (AI5), Faculty of Pharmacy, The University of Sydney, Sydney, NSW 2006, Australia

Tel +6I 29|| 40785

Fax +6I 2 935। 439।

Email rana.ahmed@sydney.edu.au 
strain family dynamics. ${ }^{10}$ Siblings of affected children can feel victimized or intimidated by the child's behaviors, while parents can find the condition emotionally taxing and timeconsuming to deal with. ${ }^{11}$ Parents of children with ADHD commonly have high levels of stress and depression, placing additional strain on family relationships. ${ }^{11}$ Approximately $50 \%$ of affected children will continue to experience such difficulties into adolescence, and of these, $30 \%-60 \%$ will continue to have impairing symptoms as adults. ${ }^{11,12}$

The current approach to treatment is multifaceted, addressing symptom control through the use of medications and psychological interventions as well as behavioral and educational strategies in order to minimize the potential long-term impacts of the disorder. ${ }^{13,14}$ The strongest evidence for improvement of ADHD symptoms is presented for prescription medications, which are effective for approximately $80 \%$ of users. ${ }^{15}$ Today, the prescribed medications for ADHD belong to two main categories; stimulants and nonstimulants. ${ }^{16}$ Stimulant medications such as methylphenidate are considered to be first-line pharmacological treatment for ADHD, ${ }^{17-19}$ while non-stimulant medications such as atomoxetine are reserved for use if a trial of stimulant medications yields no response or causes side effects. ${ }^{16}$

Despite the extensive research conducted ensuring their efficacy and safety, stimulant medications are the most contentious of the available treatment options. ${ }^{20,21}$ Media reports often portray these medications in a negative light, by raising concerns about side effects, potential for addiction, and questioning the appropriateness of medicating young children with these agents. This arouses negative public opinion and gives rise to stigmatizing attitudes towards the use of stimulant medications for ADHD treatment. ${ }^{22,23}$ Parents of affected children are consequently faced with a dilemma regarding whether or not to medicate their child, ${ }^{24}$ as media portrayals of stimulant medications often conflict with the advice and information given to them by health care professionals (HCPs) involved in their child's care. ${ }^{25}$

Therefore, it is critical that parents have access to appropriate sources of information in order to clarify any misunderstandings and address any concerns they may have about the use of prescription medications for ADHD. Access to reliable information helps parents cope with their child's illness and regain a sense of control. ${ }^{26,27}$ Information also allows parents to feel more involved in the management of their child's condition, play an empowered role in treatment decisions made, and respond to their child's questions while providing reassurance..$^{28,29}$ A recent study conducted to determine parental sources of ADHD-related information highlighted that parents rely mostly on HCPs, such as pediatricians and general practitioners (GPs), for information but also on written sources of information. ${ }^{30}$

The aim of the current study was to examine the information needs of parents of children diagnosed with ADHD, as this area has not been clearly elucidated, particularly from an Australian perspective. Specifically, we investigated the following: 1) parents' ADHD-related knowledge pre- and post-diagnosis; 2) the information sources accessed by parents, including their merits and drawbacks; 3 ) whether parents' information needs were met post-diagnosis; and 4) parents' views about strategies to meet their current and future information needs. It was the intention of the researchers to provide more insight into this area by building upon the findings of previous quantitative work and to identify where the concerns of Australian parents overlapped with or diverged from those from an international context.

\section{Methods}

\section{Focus groups}

A qualitative method, namely focus groups, was used in an attempt to gain more detailed insight ${ }^{31}$ into the parents' information needs and to build upon previous work in this area.$^{30} \mathrm{~A}$ focus group is best described as a group interview which relies on participant communication for the generation of rich data. During focus groups, participants are encouraged by the facilitator to speak to one another and to compare and contrast their experiences, anecdotes, and points of view. In this way, focus groups help not only to elicit people's knowledge about a particular topic but also how and why they arrived at this understanding. The findings presented in this paper are part of a broader investigation which also examined factors influencing parents' adherence to pharmacotherapy for ADHD. ${ }^{32}$

\section{Study participants}

\section{Inclusion criteria}

Parents were eligible to participate in the study provided that 1) their child had been diagnosed with ADHD by a clinician; 2) their child was currently taking or had (within the previous 6 months) taken one of the prescription medicines methylphenidate, dexamphetamine, or atomoxetine for ADHD; and 3) they were able to participate in the study without the assistance of a translator.

\section{Recruitment}

Parents were recruited from three separate geographic regions of the Sydney metropolitan area by a market research 
company (using their own databases of consumers) following approval from the Human Research Ethics Committee at The University of Sydney. Eighteen eligible parents were recruited, with 16 participating in the study. Each parent was reimbursed 70 AUD for their time and travel expenses.

\section{Data collection}

The focus groups were conducted in venues commonly used for focus groups, arranged by the recruitment agency and which were located in the three urban geographic areas from which the parents were initially recruited. All 16 parents provided written consent for participating in the focus groups and completed a demographic questionnaire about themselves and their child(ren) with ADHD. Participant confidentiality was stressed by the researchers, with the assurance that no demographic information would be collected on audio recordings and that all of the questionnaires and focus-group transcripts would be coded (ie, that no participant names would appear during any stage of data analysis).

The focus groups were facilitated by one of the researchers (PA) experienced in conducting focus groups, and each lasted approximately 1.0-1.5 hours. Discussions were guided by a focus-group guide, developed to address the study objectives (available from the authors on request) and were audio recorded with permission from all participants. Focus-group questions were open-ended to encourage elaboration from the participants. The focus groups were conducted until a point of saturation ${ }^{33}$ was observed in the data collected, which was at the conclusion of the third focus group.

\section{Data analysis}

Parents' focus-group responses were analyzed by two of the researchers independently, using the framework method of analysis $^{33}$ and by adopting an inductive approach. The first stage of this analysis involved familiarization with the data by listening to the recordings of each focus group several times in their entirety. These recordings were then transcribed verbatim, and the transcripts also examined multiple times while simultaneously listening to the recordings to ensure accuracy of the transcribing process. During this time, handwritten notes were made of the major themes emerging from the data, and parents' responses were compared to identify the level of agreement or disagreement within the data. The identified themes were discussed amongst three of the study authors (RA, JB, and PA) to establish the characteristics of each theme and resolve any initial differences in the identification or allocation of major and minor themes.
Once agreement about thematic identification and definition was reached, a thematic framework was developed with the assistance of the $\mathrm{NVivo}^{\circledR} 9$ (QSR International, Doncaster, VIC, Australia) qualitative data analysis software to encompass the major themes in the data and their related subtopics. Parent responses were then coded according to these major and minor themes. The identified themes and the related codes assigned to parent statements were again discussed amongst the study authors (RA, JB, and PA) to ensure consensus.

A total of three major themes and six subthemes relating to parents' information needs were identified in the data from the three focus groups. The remaining themes identified during data analysis focused on the factors influencing parents' decisions to adhere to pharmacotherapy for their children, and these are discussed elsewhere. ${ }^{32}$ Categorized information was summarized for all three focus groups, and verbatim quotations were selected to reflect the consensus of the participants following discussion amongst the study authors (RA, JB, and PA).

\section{Results}

Sixteen parents participated in the three focus groups (Table 1). The majority of the participants were male (56\%)

Table I Characteristics of focus group participants

\begin{tabular}{|c|c|}
\hline \multicolumn{2}{|c|}{ Age (missing data $=3$ ), years } \\
\hline Range & $32-55$ \\
\hline Median & 43 \\
\hline \multicolumn{2}{|l|}{ Sex, n } \\
\hline Male & 9 \\
\hline Female & 7 \\
\hline \multicolumn{2}{|c|}{ Relationship status (missing data $=2$ ), $n$} \\
\hline Single parent & I \\
\hline Married parent & 9 \\
\hline Separated parent & 3 \\
\hline Foster parent & I \\
\hline \multicolumn{2}{|l|}{ Highest education level, n } \\
\hline Primary education & 0 \\
\hline Secondary education & 6 \\
\hline Tertiary education & 7 \\
\hline Other & 3 \\
\hline \multicolumn{2}{|c|}{ Joint household income, AUD } \\
\hline $0-6,000$ & I \\
\hline $6,001-37,000$ & I \\
\hline $37,001-80,000$ & 4 \\
\hline $80,00|-| 80,000$ & 7 \\
\hline$>180,000$ & 3 \\
\hline \multicolumn{2}{|c|}{ Child's age at diagnosis (missing data $=\mathrm{I}$ ), years } \\
\hline $3-4$ & 5 \\
\hline $5-6$ & 2 \\
\hline 7-8 & 2 \\
\hline $9-10$ & 3 \\
\hline $11-12$ & 3 \\
\hline
\end{tabular}


and were the biological parents (85\%) of a child diagnosed with ADHD.

Three major themes regarding parents' ADHD-related information were identified from the data: 1) parental ADHD knowledge, 2) parental information sources, and 3) parental information needs. The parents in this study appeared to have generally limited and negative knowledge about ADHD prior to their child's diagnosis. Although their knowledge improved with time, many expressed dissatisfaction with the information they accessed, as it was technical and not tailored to the needs of their child. Most of the parents sourced verbal information from clinicians that focused on ADHD medications rather than the condition itself. Parents expressed a desire for concise, tailored information about ADHD, its medicines, and changes to their child's medication needs with age. They requested increased availability of support groups and tools to assist them in sourcing information from HCPs during consultations, such as question prompt lists (QPLs). The identified themes and subthemes are explored in greater detail below.

\section{Theme I - parental ADHD knowledge}

Prior to their child's diagnosis, the majority of parents expressed that they had very limited knowledge of ADHD. Most parents had heard about ADHD and the medication methylphenidate (Ritalin ${ }^{\circledR}$; Novartis International AG, Basel, Switzerland) through media sources, although this coverage was generally negative: "I've mainly heard about bad effects of the drugs and how children are affected" (Focus Group [FG] 1, Participant Male [M] 2).

Other parents indicated that family members or friends had raised their awareness about the condition by informing them about the possibility of their child having ADHD. Some parents worked in health-care-related fields and were familiar with the condition and its treatments, while one parent knew about the disorder through personal experience, having been affected by ADHD during childhood. Another parent indicated that his initial perception about ADHD was that "it [ADHD] was something that was just invented to keep doctors and pharmacists floating along pretty well” (FG3, M2) and was skeptical about it being an actual condition.

Generally, parents' ADHD-related knowledge improved following their child's diagnosis and stemmed from a desire to be confident about their child's diagnosis: "I did enough research to satisfy myself that I needed to accept what I was being told" (FG3, M2). However, some expressed feeling confused or frustrated by the information they received or accessed and were apprehensive about making treatment decisions as a result.

\section{Theme 2 - parental information sources}

\section{Sources of information}

The majority of the parents' primary source of ADHD-related information was the diagnosing HCP (ie, GP, pediatrician, or child psychologist/psychiatrist). This information was generally verbal. None of the parents received written information from the HCPs during consultations, although many sought such written information from the Internet: "I always go to the web and print out this [medicine information leaflet] because I like to know what [my child] is taking" (FG1, F3).

Books were another source of written information for parents. Some parents also revealed that personnel from their children's schools were able to provide them with information and support. Interestingly, no parent mentioned pharmacists as their source of medicine information. Overall, not all parents found their information sources to be satisfactory or comprehensive enough for their purposes: "I've done enough research to know that there is not enough in the information" (FG2, F2).

\section{Information covered}

There was a general consensus amongst the parents that the information they received from HCPs or from sources such as the Internet were not comprehensive and did not address all of their concerns: “... [information provided by HCP] wasn't a great deal to be honest" (FG2, F3).

The verbal information parents received from HCPs was mostly focused on the medications, including doses and side effects, rather than the condition itself. Parents sourced information from the Internet that was mostly about the experiences of other parents with children affected by ADHD and the availability of support services for parents.

\section{Problems with information accessed}

Parents noted numerous problems with the information they accessed. With regards to the verbal information from HCPs, parents explained that it was too brief (“... it was never enough. It opened up more questions than answers" [FG1, M1]) and that they often felt that the doctor was not willing to take the time to explain things with greater detail and clarity: "I would love to [tell the doctor] 'the questions I can't answer myself regarding my son are such and such, talk me through it'- I don't believe they do" (FG2, M1). Parents expressed that some HCPs not only lacked the time but also the specialized knowledge to answer their ADHD-related questions: “... our GP wasn’t familiar, wasn't confident to treat [ADHD]" (FG2, M6). Parents also felt frustrated by the conflicting advice given to them by different HCPs: "We've 
gone to a few doctors and you get a different conflicting story each time" (FG1, M1), which in turn affected the level of trust the parents had in these HCPs.

Information sought from the Internet was deemed by parents to be too excessive ("... when you have too much information, it's very hard for you to make an easy decision" [FG1, F4]), technical (“... when you look at these information leaflets, it can sort of throw you a little bit" [FG1, F2]), and most importantly, not tailored to their child's specific needs ("The trouble I have is ... this is all great information ... though ... I would sit here ... and say where does my kid fit in?" [FG2, M1]). Negative information surrounding medication side effects sought from the Internet and from HCPs was off-putting for many parents: “... the warnings ... from the doctor ... kind of freaks you out a bit" (FG1, F3), as this conflicted with the sense of urgency their HCPs had towards medicating the child: "You just get a diagnosis, you know [the child] needs [medications], but here's all the negatives about the medication" (FG1, F3). Certain information leaflets and websites were perceived to be biased by some parents if they were produced or funded by pharmaceutical companies and were consequently avoided or approached with caution: "...you're not quite sure what's driving people and whose line they're pushing" (FG3, M2).

\section{Theme 3 - parental information needs ADHD information}

Parents were very vocal about the type of information they would like to access to address their information needs. There was strong consensus amongst the parents with regards to wanting access to information in the form of recounts of real-life experiences of other parents with children affected by ADHD: “... real information about what kids were going through, rather than just the technical terms" (FG1, F3). They felt that information of this nature would be easier to relate to and that these experiences could ultimately guide them in dealing with their child's disorder and medicine-taking.

On the flipside, the parents also desired greater information about the biological aspects and causes of the disorder that was delivered clearly without the use of too much technical jargon: "The fact that there is an actual clinical reason why [ADHD develops] ... I think should be put out there more" (FG1, F1). For several parents who did have knowledge about the biological origins of the disorder, it was easier for them to appreciate how medications were going to help the child: "I had no problem putting him onto medication because [the biological causes of ADHD were] explained to me" (FG1, F1), whereas parents lacking this knowledge had difficulty accepting medications.
Parents also wanted access to information about adults who were affected by ADHD as children, especially those who used medications to treat their condition, and how their life has been affected, whether positively or negatively, as a consequence: “... research about the perspective of the drug user from the past" (FG3, F1). This long-term outlook was also reflected in the desire for parents to have more information about the long-term side effects of the medications, particularly their frequency and severity. Many parents were unsure of the duration of their child's treatment and questioned whether any changes need to be made to their treatment approach with age, especially towards adolescence: “... [the affected children] always want to know ... is there going to be an end to this [medicine-taking] or is this forever?" (FG1, F1).

Another point raised by the parents was the need for more information about their role as parents in the management of their child's ADHD ("I need to be confronted as a parent and be told what I need to do in an area that I don't have any expertise" [FG3, M2]) and how to monitor their child's progress ("I just simply don't know ... what I should be or shouldn't be flagging or diarising or charting. We don't appear to have any information on that" [FG3, M2]). A desire for easy-to-access, updated information was also expressed by the parents ("... centralising the information so everybody can be on the same page very quickly" [FG3, M2]), and the parents' general preference was for written information such as leaflets and pamphlets in conjunction with verbal information from HCPs.

\section{Support groups and skill development}

In addition to the above ADHD-related information, parents expressed a need for access to services which would allow them to develop skills and techniques that would assist in the management of their child's condition. Parents suggested that the diagnosing HCP should refer them to support groups to allow them to witness first-hand what other parents were going through, particularly with regards to the impacts of medicating or not medicating the affected child: "If you had a problem, I reckon that support groups would be more help than any books or pamphlets" (FG1, M1). There was also a strong desire amongst parents to be taught strategies they could use to identify and deal with their child's mood swings as well as general home practices to help them respond to the child's needs in general: "I would like to see a little bit of action regarding positive identification of mood swings, what to do in certain situations" (FG2, M1). Parents wanted help in setting tailored goals for their child to allow them to gauge 
their child's progress: “... having some goals or objectives that I should be looking for at certain points, nailed down to my son's particular situation" (FG3, M2).

Parents also wanted guidance about other issues following their child's diagnosis, including whether or not they needed to contact and inform certain personnel in the child's school or in the education department about their child's condition: "When your son or daughter is diagnosed ... you go to school, you tell the special needs teachers ... make applications with the education department, all that sort of stuff ... you just don't think of it really" (FG1, M1). One parent felt that this should be the responsibility of the diagnosing HCP: "I think it's up to [the doctor] to follow [diagnosis] through ... to send [information] through to the [child's] teacher [addressing] specific questions" (FG1, M1).

\section{Question prompt lists}

During the first focus-group session, parents suggested an idea of having a list of questions to take in with them during consultations as a way of addressing their desire for written information tailored to their child's needs: "I really like the idea of having a list of questions ..." (FG1, M2). In the literature, this tool is best described as a QPL, and this idea was well received during the initial focus group and in subsequent focus groups when parents were questioned about their thoughts about such a resource. Parents described that by taking a tailored list of questions with them during consultations with HCPs, they would be able to obtain relevant information from the HCP without being overloaded by information: "When you get the information overload, at first you are in shock ... That [QPL] would be good" (FG1, F2). The parents also felt that by having this list, they would be able to address their concerns more effectively, without forgetting to ask any potentially important questions. The list would also allow them to write down the answers to their questions, and this written information could be kept by the parents for future reference and in the case that they forgot any verbal information given to them by the HCP: “... it would be so convenient to have that" (FG2, M1).

\section{Discussion}

Although previous quantitative work has been conducted examining parents' views about sources of information and their information needs, ${ }^{30}$ there remained a need for more indepth investigation in this area through qualitative inquiry. This study builds on the findings of such studies by providing further insight into and a richer understanding of the specific preferences and needs of parents, thereby addressing this gap in the literature. Further, this is the first study to identify the concept of a QPL as a potentially important and useful strategy in addressing the ADHD-related information needs of parents.

Parents' knowledge of ADHD prior to their child's diagnosis was mostly confined to an awareness of methylphenidate $\left(\right.$ Ritalin $^{\circledR}$ ) gained through negative media reports and public opinion. This finding is consistent with previous studies which reveal that members of the public are not well informed about ADHD or its medicines and that the information that they do have is generally negative. ${ }^{34}$ Public knowledge and individuals' opinions about ADHD are complex issues, which have been shown in previous work to be dependent upon ethnicity, age, and sex, for example. ${ }^{34}$ What is apparent, however, is that there is a level of public resistance to a biomedical conceptualization of ADHD and consequently, its treatment using medications. ${ }^{35}$ It is this resistance that either encourages or is encouraged by negative media reports which, when coupled with minimal or inadequate public awareness about $\mathrm{ADHD}$, gives rise to misinformation about the disorder and its treatments. Although the parents' ADHD-related knowledge improved following their child's diagnosis, many parents expressed dissatisfaction with the information sources they accessed and vocalized a feeling of overall confusion with the information obtained from them, a notion not identified in previous quantitative work.

The majority of the parents in this study accessed multiple sources of information about ADHD, highlighting the extent of parents' desire for information. The primary source of information for the parents was the diagnosing HCP, including GPs, pediatricians, and child psychologists, which is consistent with the findings of previous research. ${ }^{30}$ Further to these findings, however, were the specific concerns expressed by the parents in this study about the nature of the information sourced from these HCPs. Verbal information from some HCPs was viewed to be too brief, incomprehensive, and at times, contradicted with information issued by other HCPs. It is interesting to note that despite HCPs being a common source of information for the parents in this study, they were not necessarily perceived to provide relevant information. These findings are in contrast to those of previous work, where HCPs were deemed to be both the most commonly accessed and most easy to understand information source. ${ }^{30}$ A potential justification for this point of difference is that the qualitative nature of this work allowed parents to voice concerns in a more in-depth and open fashion, as opposed to the use of surveys observed in the latter study, which may have restricted parents' ability to discuss the specifics of their interactions with HCPs. Thus, the fact that HCPs 
are a popular source of information for parents does not discount from the fact that their approach to information delivery may require improvement.

This finding is corroborated by previous studies of other chronic conditions which reveal low parental satisfaction with the quality of information issued by $\mathrm{HCPs},{ }^{36,37}$ and an inability of some HCPs to adequately communicate information. ${ }^{37}$ Ideally, the information sourced from the HCP should be clear and reliable. However, parents often receive inaccurate information, insufficient or excessive information, and information that is not in line with their specific concerns. ${ }^{36}$ Such inadequacy in communication between parents and clinicians can ultimately lead to misconceptions about the condition, inability to express treatment preferences, and poor adherence to treatment programs. ${ }^{37}$ The observed disparity between the amount, quality, and consistency of information desired by parents and that delivered to them can be attributed to several factors. Studies highlight that HCPs often do not meet the information needs of parents as they underestimate the parent's desire for information, have limited consultation times, and may lack the appropriate knowledge of a particular condition. ${ }^{37,38}$

Information sourced from the Internet also had its drawbacks, with the main parental concern being the excessive amount of information available to them, in addition to concerns about the reliability of this information. This is consistent with the findings of a previous study which highlights that while parents may use the Internet to find information, they do not necessarily trust this information. ${ }^{30}$ Information found on the Internet was also perceived to be too general and did not address the specific concerns of parents in this study.

The specific information needs as expressed by the parents in this study provide avenues for future research. Parents strongly supported the idea of having access to information to which they could more easily relate, for example real-life experiences of other families in the same situation. As the information that parents sourced from HCPs was mostly focused on the medications used to treat ADHD, parents also wanted more information about the biological causes of the disorder, as this was not clear for many parents, particularly those who questioned whether their parenting skills played a role in their child's development of the disorder.

Parents also requested more information about their role as parents in the management of their child's ADHD, reinforcing the understanding that the management of ADHD is complex and multifaceted, not only involving medications, but also behavioral and educational interventions and strategies. This finding is in line with previous work concerning parents of children with $\mathrm{ADHD}^{30}$ and learning disabilities. ${ }^{39}$ In addition to this, the parents in the present study also expressed a strong need for access to support groups to which they can be referred by their HCPs, as many expressed that they had great difficulty in locating such services on their own. This interest in the experiences and firsthand knowledge of other parents also reinforces the notion that parents expect information provision to be a continuous, personalized process rather than a one-off event. ${ }^{26,39}$

Overall, in this study, parents expressed a preference for written information such as leaflets and pamphlets to be used alongside verbal information from HCPs. This is supported by previous work, which revealed that $80 \%$ of parents would like to access ADHD information in written form and that $77 \%$ would like verbal information. ${ }^{30}$ The qualitative nature of this investigation, however, permitted a more in-depth examination of parents' specific requests about the nature of the written information they wished to receive. A unique finding of this investigation was parents' interest in the concept of a QPL as a written resource to allow them to obtain relevant information from HCPs.

The use of QPLs is a relatively novel approach devised to assist patients in shared decision-making processes with their HCPs. QPLs contain a structured list of questions that patients may choose to ask their physician about their illness and its treatment, and have been shown to enhance physician-patient communication. ${ }^{40,41}$ Questions are usually derived from the analyses of physician-patient interactions and/or focus groups with both parties. ${ }^{41}$ Traditionally, the tool has been used in oncology care settings and has proven to be an inexpensive and effective facilitator for communication during consultations. ${ }^{42,43}$ In this setting, QPLs have been shown to increase the number of questions asked by both patients and their caregivers, particularly about difficult topics such as prognosis, without increasing anxiety. ${ }^{43}$ QPLs also give patients or their carers ideas about questions to ask during consultations, which is especially useful for those receiving a new diagnosis and who may lack basic understanding of the condition and its treatment. Importantly, QPLs allow patients to have control over the information they receive from the clinician (ie, they can direct the focus of information provision). Furthermore, a recent meta-analysis comparing decision support tools concluded that QPLs are at least as effective, far cheaper, and more acceptable as decision aids. ${ }^{44}$

The concept of QPLs has been utilized in a range of other diseases, including diabetes and with parents of children 
with neurological problems, and may thus prove useful in empowering parents of children with ADHD to source relevant information from their child's clinician. Utilizing this tool would assist in addressing the inability of parents to ask the right questions during consultations ${ }^{29}$ and their desire to use written resources as a prompt for communication with their child's HCP. ${ }^{37}$ As HCPs are a popular source of information for parents, the use of a QPL has the potential to facilitate better communication and improve parental understanding of verbal information. Importantly, this list of questions would ensure that parents are obtaining their information from a reliable source while at the same time allowing parents to access information that is relevant to the needs of their child at a specific point in time. This may help to minimize the difficulty and ambivalence parents commonly experience when gathering information about treatments ${ }^{25,45}$ and empower them to play a more active role in their child's health.

\section{Limitations}

The findings of this investigation should be viewed in light of certain limitations. Firstly, the qualitative nature of this study means that no specific hypotheses were formulated and tested. Rather, the researchers' use of a qualitative approach was intended to allow for more in-depth insight ${ }^{46}$ into parents' opinions about their first-hand experiences with ADHD and their information needs, while building upon the findings of previous quantitative work. Considering that this area of investigation remains underreported and not well understood, especially from an Australian perspective, this qualitative approach in many ways was the most rigorous and appropriate way to establish insights into this topic. ${ }^{47}$ This is demonstrated in the novel findings of the current study, including parents' strong support for the development of tools such as QPLs to help them meet their information needs, which may have otherwise been difficult to elicit. Therefore, although this qualitative investigation may not be generalizable to the entire population, the rich data collected can be used as the basis of future quantitative investigations. For example, the findings of this exploratory study can be used to develop interventions targeted at parents of children with ADHD to promote access to information and address issues surrounding suboptimal adherence to therapy. ${ }^{32,48}$

The intentional decision to use focus groups in favor of interviews was made in anticipation that the parents' ability to relate to or contrast with the experiences of other parents would stimulate discussion and encourage participants to voice their opinions. Care was taken by the focus group facilitator to ensure that each parent was able to contribute to the same extent as other potentially more vocal parents during the discussions.

The parents involved in this study were recruited from metropolitan Sydney areas only, which may affect the generalizability of the study findings to other populations. In addition, only a small number of participants $(n=16)$ were needed to reach data saturation. Although the findings of this qualitative study are not necessarily intended to be generalized to the entire population, there was a strong level of agreement between the findings of the present study and those conducted previously, supporting their relevance to this area of the literature.

\section{Conclusion}

Exploring parents' information needs more broadly and using a qualitative approach provided a more in-depth understanding of their specific needs and preferences and built upon the findings of previous quantitative studies. Most parents in this study obtained information from multiple sources, including verbal information from HCPs and written information as found in books and on the Internet. The parents highlighted that there are flaws with each source and that they often felt unsupported and isolated as a consequence of not being able to access appropriate information for their purposes. The novel concept of a QPL for ADHD was a well-received parental suggestion, with parents expressing that it would help them obtain relevant information from a reliable source that could be updated with time. The development and testing of this tool is the next phase of this research. It is essential that future research focuses on providing parents with alternative avenues to access relevant and reliable information and support in order to empower parents to make the best decision for their child.

\section{Acknowledgment}

The authors would like to thank all of the parents involved in this study.

\section{Disclosure}

The authors report no conflicts of interest in this work. The authors received no financial support for the research, authorship, and/or publication of this article.

\section{References}

1. Curatolo P, D'Agati E, Moavero R. The neurobiological basis of ADHD. Ital J Pediatr. 2010;36(1):79.

2. Braaten EB, Rosén LA. Self-regulation of affect in attention deficithyperactivity disorder (ADHD) and non-ADHD boys: differences in empathic responding. J Consult Clinl Psych. 2000;68(2):313-321.

3. Daley D, Birchwood J. ADHD and academic performance: why does ADHD impact on academic performance and what can be done to support ADHD children in the classroom? Child Care Health Dev. 2009;36(4): 455-464. 
4. American Psychiatric Association. Diagnostic and statistical manual of mental disorders, Fourth Edition, Text Revision. Washington, DC: American Psychiatric Association; 2000.

5. Polderman TJC, Boomsma DI, Bartels M, Verhulst FC, Huizink AC. A systematic review of prospective studies on attention problems and academic achievement. Acta Psychiatr Scand. 2010;122:271-284.

6. Rowles BM, Findling RL. Review of pharmacotherapy options for the treatment of attention-deficit/hyperactivity disorder (ADHD) and ADHD-like symptoms in children and adolescents with developmental disorders. Dev Disabil Res Rev. 2010;16:273-282.

7. Lien MT, Carlson JS, Hunter-Oehmke S, Knapp KA. A pilot investigation of teachers' perceptions of psychotropic drug use in schools. J Attention Disord. 2007;11(2):172-178.

8. Culpepper L. Primary care treatment of attention-deficit/hyperactivity disorder. J Clin Psychiat. 2006;67(Suppl 8):51-58.

9. DeNisco S, Tiago C, Kravitz C. Evaluation and treatment of pediatric ADHD. Nurse Pract. 2005;30(8):14-23.

10. Sawyer MG, Whaites L, Rey JM, Hazell PL, Graetz BW, Baghurst P. Health related quality of life of children and adolescents with mental disorders. J Am Acad Child Adolesc Psychiatry. 2002;41(5):530-537.

11. Salmeron PA. Childhood and adolescent attention-deficit hyperactivity disorder: diagnosis, clinical practice guidelines and social implications. J Am Acad Nurse Pract. 2008;21:488-497.

12. Barkley RA. Attention Deficit Hyperactivity Disorder: A Handbook for Diagnosis and Treatment. New York: Guildford Press; 1990.

13. Knutson KC, O’Malley M. Adult attention-deficit/hyperactivity disorder: a survey of diagnosis and treatment practices. J Am Acad Nurse Pract. 2009;22:593-601.

14. Murray DW. Treatment of preschoolers with attention-deficit/ hyperactivity disorder. Curr Psychiatry Rep. 2010;12:374-381.

15. Faraone SV, Biederman J, Spencer TJ, Aleardi M. Comparing the efficacy of medications for ADHD using meta-analysis. Med Gen Med. 2006;8(4):4.

16. Wigal SB, Chae S, Patel A, Steinberg-Epstein R. Advances in the treatment of attention-deficit/hyperactivity disorder: a guide for pediatric neurologists. Semin Pediatr Neurol. 2010;17:230-236.

17. Jadad AR, Booker L, Gauld M, et al. The treatment of attention-deficit hyperactivity disorder: An annotated bibliography and critical appraisal of published systematic reviews and metaanalyses. Can J Psychiat 1999;44(10):1025-1035.

18. Kaplan G, Newcorn JH. Pharmacotherapy for child and adolescent attention-deficit hyperactivity disorder. Pediatr Clin North Am. 2011;58: 99-120.

19. Singer-Leshinsky S. Attention-deficit/hyperactivity disorder: helping families to achieve success. JAAPA. 2011;24(3):52-57.

20. Bussing R, Gary FA. Practice guidelines and parental ADHD treatment evaluations: friends or foes? Harvard Rev Psychiat. 2001;9:223-233.

21. Daley D. Attention deficit hyperactivity disorder: a review of the essential facts. Child Care Health Dev. 2006;32(2):193-204.

22. Olaniyan $\mathrm{O}$, dosReis $\mathrm{S}$, Garriett $\mathrm{V}$, et al. Community perspectives of childhood behavioral problems and ADHD among African American parents. Ambul Pediatr. 2007;7(3):226-231.

23. Peters K, Jackson D. Mothers' experiences of parenting a child with attention deficit hyperactivity disorder. J Adv Nurs. 2009;65(1):62-71.

24. Ahmed R, McCaffery KJ, Aslani P. Factors influencing parental decision-making about stimulant treatment for attention deficit hyperactivity disorder. J Child Adolesc Psychopharmacol. 2013;23(3): 163-178.

25. McGuinness TM. Helping parents decide on ADHD treatment for their children. J Psychosoc Nurs Ment Health Serv. 2008;46(8):23-27.

26. Sloper P. Service needs of families of children with severe physical disability. Child Care Health Dev. 1992;18:259-282.

27. Starke M, Möller A. Parents' needs for knowledge concerning the medical diagnosis of their children. J Child Health Care. 2002;6(4): 245-257.
28. Davies S, Hall D. "Contact a family": professionals and parents in partnership. Arch Dis Child. 2005;90:1053-1057.

29. Hummelinck A, Pollock K. Parents' information needs about the treatment of their chronically ill child: a qualitative study. Patient Educ Couns. 2006;62(2):228-234.

30. Sciberras E, Iyer S, Efron D, Green J. Information needs of parents of children with attention-deficit/hyperactivity disorder. Clin Pediatr. 2010;49(2):150-157.

31. Morton RL, Tong A, Howard K, Snelling P, Webster AC. The views of patients and carers in treatment decision making for chronic kidney disease: systematic review and thematic synthesis of qualitative studies. BMJ. 2010;340:c112.

32. Ahmed R, Borst J, Wei YC, Aslani P. Parents' perspectives about factors influencing adherence to pharmacotherapy for ADHD. J Atten Disord. Epub August 30, 2013.

33. Cameron J. Focusing on the focus group. In: Hay I, editor. Qualitative Research Methods in Human Geography. Melbourne: Oxford University Press; 2005:156-182.

34. McLeod JD, Fettes DL, Jensen PS, Pescosolido BA, Martin JK. Public knowledge, beliefs, and treatment preferences concerning attentiondeficit hyperactivity disorder. Psych Serv. 2007;58(5):626-631.

35. Summers JA, Caplan PJ. Laypeople's attitudes toward drug treatment for behavioural control depend on which disorder and which drug. Clin Pediatr. 1987;26:258-263.

36. Fisher HR. The needs of parents with chronically sick children: a literature review. J Adv Nurs. 2001;36(4):600-607.

37. Coulter A, Entwistle V, Gilbert D. Sharing decisions with patients: is the information good enough? BMJ. 1999;318:318-322.

38. McConkey R. Information needs of parents about learning disabilities. J Learn Disabil. 2003;7:211-219.

39. Pain H. Coping with a child with disabilities from the parents' perspective: the function of information. Child Care Health Dev. 1999;25(4):299-312.

40. Caminiti C, Diodati F, Filiberti S, et al. Cross-cultural adaptation and patients' judgments of a question prompt list for Italian-speaking cancer patients. BMC Health Serv Res. 2010;10:16.

41. Dimoska A, Tattersall MHN, Butow PN, Shepherd H, Kinnersley P. Can a "prompt list" empower cancer patients to ask relevant questions? Cancer. 2008;113(2):225-237.

42. Clayton J, Butow P, Tattersall M, et al. Asking questions can help: development and preliminary evaluation of a question prompt list for palliative care patients. Br J Cancer. 2003;89:2069-2077.

43. Clayton JM, Butow PN, Tattersall MHN, et al. Randomized controlled trial of a prompt list to help advanced cancer patients and their caregivers to ask questions about prognosis and end of life care. J Clin Oncol. 2007;25(6):715-723.

44. Spiegle G, Al-Sukhni E, Schmocker S, et al. Patient decision aids for cancer treatment: are there any alternatives? Cancer. 2013;119(1): 189-200.

45. Bennet DS, Power TJ, Rostain AL, Carr DE. Parent acceptability and feasibility of ADHD interventions: assessment, correlates and predictive validity. J Pediatr Psychol. 1996;21(5):643-657.

46. Powell RA, Single HM. Focus groups. Int J Qual Health Care. 1996; $8(5): 499-504$.

47. Giacomini MK, Cook DJ. Users' guides to the medical literature: XXIII. Qualitative research in healthcare A: are the results of the study valid? Evidence-Based Medicine Working Group. JAm Med Assoc. 2000;248: $357-362$.

48. Ahmed R, Aslani P. Attention-deficit/hyperactivity disorder: an update on medication adherence and persistence in children, adolescents and adults. Expert Rev Pharmacoecon Outcomes Res. 2013;13(6): $791-815$. 
Patient Preference and Adherence

Dovepress

\section{Publish your work in this journal}

Patient Preference and Adherence is an international, peer-reviewed, open access journal focusing on the growing importance of patient preference and adherence throughout the therapeutic continuum. Patient satisfaction, acceptability, quality of life, compliance, persistence and their role in developing new therapeutic modalities and compounds to

optimize clinical outcomes for existing disease states are major areas of interest. This journal has been accepted for indexing on PubMed Central. The manuscript management system is completely online and includes a very quick and fair peer-review system. Visit http://www.dovepress.com/ testimonials.php to read real quotes from published authors.

Submit your manuscript here: http://www.dovepress.com/patient-preference-and-adherence-journal 